\title{
Regional Beta Index of Electroencephalography May Differentiate Alzheimer's Disease from Depression
}

\author{
Kanghee Lee ${ }^{1}$, Ji Won Han', and Ki Woong Kim,2,3凶 \\ ${ }^{1}$ Department of Neuropsychiatry, Seoul National University Bundang Hospital, Seongnam, Republic of Korea \\ ${ }^{2}$ Department of Psychiatry, Seoul National University College of Medicine, Seoul, Republic of Korea \\ ${ }^{3}$ Department of Brain and Cognitive Science, Seoul National University College of Natural Sciences, Seoul, Republic of Korea
}

Differentiating early Alzheimer's disease (AD) from depression with cognitive impairment is challenging in the elderly. To develop a model for differentiating these two conditions using electroencephalography (EEG), we enrolled 11 patients with early probable AD and 11 age- and cognitive function-matched patients with major depressive disorder (MDD) and compared the EEG relative powers of 9 scalp regions. Compared to the $\mathrm{MDD}$ group, the $\mathrm{AD}$ group had a higher global theta relative power ( $\mathrm{p}=0.021)$. In the MDD group, beta relative power was higher in the mid-central region than in the left or right central regions $(\mathrm{p}<0.01)$. The prediction model that included global theta relative power and regional beta index was able to discriminate $\mathrm{AD}$ from $\mathrm{MDD}$ ( $\mathrm{AUC}=0.893, \mathrm{p}=0.002$ ). A combination of global theta relative power and intra-individual regional differences in beta may differentiate early AD from MDD with cognitive impairment.

Psychiatry Investig 2017;14(5):708-711

Key Words Alzheimer's disease, Major depressive disorder, Differential diagnosis, EEG, Theta, Beta.

\section{INTRODUCTION}

In the elderly, differentiation of cognitive impairment due to Alzheimer's disease (AD) from that of depression is one of the major challenges in both clinical and research settings. ${ }^{1}$ Dementia and depression are common and frequently comorbid in the elderly. ${ }^{2}$ Depressive symptoms are associated with poor cognitive function and rapid cognitive decline with advancing age. ${ }^{3}$ However, there are no biological markers that can successfully discriminate early $\mathrm{AD}$ from cognitive impairment associated with depressive disorders in the elderly. Previous studies indicate that changes in electroencephalography (EEG) associated with $\mathrm{AD}$ may be different from those associated with depressive disorders. ${ }^{4-10}$ Compared to normal controls, patients with $\mathrm{AD}$ had increases in slow waves and decreases in fast waves in EEG. ${ }^{4-7}$ On the other hand, patients with major depressive disorder (MDD) had increased alpha activity and

Received: July 14, 2016 Revised: September 12, 2016

Accepted: November 19, 2016 Available online: July 26, 2017

$\triangle$ Correspondence: Ki Woong Kim, MD, PhD

Department of Neuropsychiatry, Seoul National University College of Medicine and Seoul National University Bundang Hospital, 82 Gumi-ro 173 beon-gil, Bundang-gu, Seongnam 13620, Republic of Korea

Tel: +82-31-787-7432, Fax: +82-31-787-4058, E-mail: kwkimmd@snu.ac.kr

(a) This is an Open Access article distributed under the terms of the Creative Commons Attribution Non-Commercial License (http://creativecommons.org/licenses/bync/4.0) which permits unrestricted non-commercial use, distribution, and reproduction in any medium, provided the original work is properly cited. asymmetric alpha activity in frontal regions. ${ }^{9}$ However, thus far only a few studies have directly compared the EEG spectrum in both patients with $\mathrm{AD}$ and those with depressive disorders. Furthermore, there are no reports describing consistent common features that may be used to differentiate $\mathrm{AD}$ from depressive disorders. In this study, we directly compared EEG activity in patients with $\mathrm{AD}$ without depression with that in age- and cognitive function-matched patients with MDD. We adjusted for structural brain differences using brain magnetic resonance imaging (MRI) and found EEG features that successfully differentiated these two conditions.

\section{METHODS}

The research protocol was reviewed and approved by the Seoul National University Bundang Hospital (SNUBH) Institutional Review Board (IRB No: B-1610-366-108). The subjects included 11 patients with probable $\mathrm{AD}$ (7 females), who were diagnosed according to the National Institute of Neurological and Communicative Disorders and Stroke and Alzheimer's Disease and Related Disorders Association diagnostic criteria. ${ }^{11}$ Our study also included 11 age- and Short Blessed Test (SBT) score-matched patients with MDD (9 females), who were diagnosed according to the 4th edition of Diagnostic and Statistical Manual of Mental Disorders diagnostic criteria. The 
SBT, originally designed to identify dementia, consists of six items to evaluate orientation, registration or attention. ${ }^{12,13}$ Our subjects were selected from elderly Koreans aged 60 years or older who had visited the Dementia Clinic of SNUBH from 2012 to 2013 with complaints of cognitive decline. The Clinical Dementia Ratings (CDR) of the patients with AD were 1 or 0.5 when they undergo the clinical assessment and EEG motoring. Subjects with comorbid neurologic or psychiatric disorders that may have influenced EEG activity and those taking antiepileptic or mood-stabilizing medications were excluded.

All subjects underwent a standardized clinical assessment within a month of their EEG motoring, using the Korean version of the Consortium to Establish a Registry for Alzheimer's Disease Assessment Battery (CERAD-K) ${ }^{14}$ and the Mini International Neuropsychiatric Interview (MINI) ${ }^{15}$ The subjects also completed the Korean version of the Geriatric Depression Scale (GDS-K) ${ }^{16}$ by themselves. We obtained brain MRIs from the subjects, and based on the agreements, 4 geriatric psychiatrists who blinded to the subjects' EEG results graded the following 3 indices to adjust the influence of structural brain asymmetry on EEG activity: medial temporal atrophy (MTA) score, ${ }^{17}$ Koedam score for the posterior atrophy, ${ }^{18}$ and Fazekas scores for the white matter hyperintensities (WMH). ${ }^{19}$

Resting-state EEGs were recorded for 15 minutes ( 7 minutes with eyes closed, 1 minute with eyes open, and 7 minutes with eyes closed) by using a 64-channel quick-cap with a Neuroscan SynAmp2 amplifier (Compumedics, Victoria, Australia). The EEG data were transformed in computer-averaged mastoids (M1 and M2) in an offline analysis. Twelve 10-second closedeye noise-free epochs were prepared for each subject using a manual screen. Fast Fourier transformation (FFT) was performed on 62 electrodes split into 5 frequency bands (delta, 1-3 Hz; theta, 4-7 Hz; alpha, 8-12 Hz; beta, $13-25 \mathrm{~Hz}$; and gamma, $30-50 \mathrm{~Hz}$ ) and 2 sub-bands of alpha and beta (alpha1, 8-10 Hz; alpha2, 11-12 Hz; beta1, 13-20 Hz; and beta2, 21$25 \mathrm{~Hz}$ ). To calculate the relative global band powers, we followed the method of previous studies. ${ }^{4,7,20} \mathrm{~A}$ total of 9 regions were selected based on previous EEG study ${ }^{21}$ for further analysis: left-frontal, mid-frontal, right-frontal, left-central, midcentral, right-central, left-posterior (parieto-occipital), mid-posterior, and right-posterior.

Repeated measures multivariate analysis of variance (MANOVA) was performed to compare EEG relative power in the $\mathrm{AD}$ and MDD groups. The polarity (frontal, central, and posterior) and laterality of the electrodes (left, mid, and right) were computed as within-subject variables. To evaluate the efficacy of EEG as a diagnostic tool for discriminating AD from MDD, a stepwise logistic regression analysis was performed with EEG parameters that were significantly different between groups in the former analyses.

\section{RESULTS}

There were no significant differences in the demographics (age, sex, and education level) and the 3 indices of brain structure between the AD and MDD groups. Patients in the MDD group had higher GDS scores than those in the AD group ( $\mathrm{p}=$ 0.014) (Table 1). The repeated measures MANOVA analysis indicated that the $\mathrm{AD}$ group had higher global power in the theta band than the MDD group (17.43 \pm 10.41 vs. $9.168 \pm 8.84$, $\mathrm{F}=6.302, \mathrm{p}=0.021$ ). In addition, the 2 -way interaction between the laterality and the diagnostic group was significant in the beta $(\mathrm{F}=4.19, \mathrm{p}=0.031)$ and beta1 $(\mathrm{F}=4.145, \mathrm{p}=0.027)$ bands. In the MDD group, the relative powers of the beta, betal, and beta2 bands were higher in the mid-central region compared to the left-central or right-central regions $(\mathrm{p}<0.01)$. They were also higher in the mid-frontal region compared to the left-frontal region $(\mathrm{p}<0.05)$. However, such regional differences in the beta were not observed in the $\mathrm{AD}$ group.

To develop the score representing the regional differences of beta power, we calculated the regional beta index (RBI) as follows: RBI=[Beta_MC-(Beta_LC+Beta_RC)/2], where Beta MC, Beta_LC, and Beta_RC denote the beta relative power in the mid-central, left-central, and right-central regions, respectively. Global theta power and RBI were used as independent variables and the diagnostic group ( $\mathrm{AD}$ versus $\mathrm{MDD}$ ) was used as a dependent variable in the stepwise regression analysis. Global theta power and RBI were included in the final predictive model for differentiating early $\mathrm{AD}$ from MDD with cognitive impairment $\left(\chi^{2}=12.00, \mathrm{p}=0.002\right)$. The prediction ability of

Table 1. Demographic and clinical characteristics of the subjects

\begin{tabular}{lccc}
\hline & $\mathrm{AD}$ & $\mathrm{MDD}$ & \\
& $(\mathrm{N}=11)$ & $(\mathrm{N}=11)$ & $\mathrm{p}^{*}$ \\
\hline Age (years, mean $\pm \mathrm{SD})$ & $70.8 \pm 9.2$ & $69.4 \pm 9.2$ & 0.622 \\
Education (years, mean $\pm \mathrm{SD})$ & $11.3 \pm 4.0$ & $6.6 \pm 5.1$ & 0.054 \\
Sex (female, \%) & 63.6 & 81.8 & 0.635 \\
GDS (points, mean $\pm \mathrm{SD})$ & $14.8 \pm 6.6$ & $22.7 \pm 6.6$ & 0.014 \\
SBT (points, mean $\pm \mathrm{SD}$ ) & $12.3 \pm 6.0$ & $9.6 \pm 6.2$ & 0.277 \\
Medial temporal atrophy score & & & \\
$\quad$ Left (score, mean $\pm \mathrm{SD}$ ) & $1.9 \pm 0.70$ & $1.7 \pm 1.03$ & 0.215 \\
$\quad$ Right (score, mean $\pm \mathrm{SD})$ & $2.09 \pm 0.94$ & $1.18 \pm 1.08$ & 0.069 \\
Koedam score & & & \\
$\quad$ Left (score, mean $\pm \mathrm{SD}$ ) & $1.1 \pm 0.7$ & $0.82 \pm 0.75$ & 0.372 \\
$\quad$ Right (score, mean $\pm \mathrm{SD}$ ) & $0.91 \pm 0.54$ & $0.82 \pm 0.75$ & 0.748 \\
Fazekas score & & & \\
$\quad$ PVWM (score, mean $\pm \mathrm{SD})$ & $1.1 \pm 0.9$ & $0.7 \pm 0.8$ & 0.363 \\
$\quad$ DWM (score, mean $\pm \mathrm{SD})$ & $1.2 \pm 1.0$ & $0.9 \pm 0.8$ & 0.534 \\
\hline
\end{tabular}

*Mann-Whitney's U-test or Fisher's exact test. AD: Alzheimer's disease, MDD: major depressive disorder, GDS: Geriatric Depression Scale, SBT: Short Blessed Test, PVWM: periventricular white matter, DWM: deep white matter 
this model was better $\left(\chi^{2}=5.84, \mathrm{p}=0.016\right)$ than that of a model including only global theta power $\left(\chi^{2}=6.16, p=0.013\right)$. According to receiver operating characteristic curve analysis, the area under the curve (AUC) of the model that included global theta power alone was $0.793(\mathrm{p}=0.020)$ while that of the model that included both global theta power and RBI was much better (AUC=0.893, $\mathrm{p}=0.002$ ).

\section{DISCUSSION}

In this study, patients with $\mathrm{AD}$ had higher theta wave activities over all scalp areas. In addition, patients in the $\mathrm{AD}$ group did not have intra-individual regional differences in beta power, as was observed in the central regions in patients with MDD. The model used to differentiate $\mathrm{AD}$ from MDD with cognitive impairment had good discrimination (AUC=0.893).

Increased theta activity had been reported to be a pathognomonic EEG feature of $\mathrm{AD}{ }^{7,22} \mathrm{AD}$ patients had higher theta relative power than MDD patients in both the resting state 2 and in the REM-sleep state. ${ }^{23}$ In addition, in patients with $\mathrm{AD}$, theta activity had a strong negative correlation with performance in the Test for the Early Detection of Dementia with Discrimination from Depression. ${ }^{24}$ In line with these studies, global theta power was a principal predictor in discriminating $\mathrm{AD}$ from MDD with cognitive impairment in the current study. However, the accuracy of this predictor was limited as a diagnostic marker (ACU=0.793).

Interestingly, in patients with MDD, the relative powers of the beta bands were stronger in the midline areas than in the left or right areas, particularly in the central regions. These intra-individual differences in beta relative power, however, were not observed in patients with $\mathrm{AD}$. When we employed the RBI that reflects this hyper beta activity in the mid-central area compared to the right or left central areas in combination with global theta power in the predictive model for discriminating $\mathrm{AD}$ from MDD with cognitive impairment, the AUC of the model significantly improved to 0.893 . Ihl and Brinkmeyer ${ }^{10}$ reported that patients with MDD had more distinct beta segments than patients with $\mathrm{AD}$, as indicated by spaceoriented adaptive segmentation. A segment is a stable topographic distribution of the EEG signal over a period of time. The intra-individual regional differences in beta relative power in patients with MDD in the current study may be related to the higher number of such segments.

In this study, we rigorously controlled potential confounding factors (age, sex, education, cognitive function, and structural abnormalities of the brain) in both patients with $\mathrm{AD}$ and those with MDD. However, our observations warrant further investigation, as our sample size was small and the use of medications was not controlled for.

\section{Acknowledgments}

This work was supported by a grant from the Korean Health Technology R\&D Project, Ministry of Health and Welfare, Republic of Korea [grant number HI09C1379 (A092077)].

\section{REFERENCES}

1. Dykierek P, Stadtmuller G, Schramm P, Bahro M, van Calker D, Braus DF, et al. The value of REM sleep parameters in differentiating Alzheimer's disease from old-age depression and normal aging. J Psychiatr Res 1998;32:1-9.

2. Deslandes A, Veiga H, Cagy M, Fiszman A, Piedade R, Ribeiro P. Quantitative electroencephalography (qEEG) to discriminate primary degenerative dementia from major depressive disorder (depression). Arq Neuropsiquiatr 2004;62:44-50.

3. Dotson VMP, Resnick SMP, Zonderman ABP. Differential association of concurrent, baseline, and average depressive symptoms with cognitive decline in older adults. Am J Geriatr Psychiatry 2008;16:318-330.

4. Kim JS, Lee SH, Park G, Kim S, Bae SM, Kim DW, et al. Clinical implications of quantitative electroencephalography and current source density in patients with Alzheimer's disease. Brain Topogr 2012;25: 461474.

5. Prichep LS. Quantitative EEG and electromagnetic brain imaging in aging and in the evolution of dementia. Ann N Y Acad Sci 2007;1097: 156-167.

6. Roh JH, Park MH, Ko D, Park KW, Lee DH, Han C, et al. Region and frequency specific changes of spectral power in Alzheimer's disease and mild cognitive impairment. Clin Neurophysiol 2011;122:2169-2176.

7. Jung HT, Lee SH, Kim JN, Lee KJ, Chung YC. Quantitative electroencephalography and low resolution electromagnetic tomography imaging of Alzheimer's disease. Psychiatry Investig 2007;4:31-37.

8. Knott V, Mohr E, Mahoney C, Ilivitsky V. Quantitative electroencephalography in Alzheimer's disease: comparison with a control group, population norms and mental status. J Psychiatry Neurosci 2001;26: 106-116.

9. Olbrich S, Arns M. EEG biomarkers in major depressive disorder: discriminative power and prediction of treatment response. Int Rev Psychiatry 2013;25:604-618.

10. Ihl R, Brinkmeyer J. Differential diagnosis of aging, dementia of the Alzheimer type and depression with EEG-segmentation. Dement Geriatr Cogn Disord 1999;10:64-69.

11. McKhann G, Drachman D, Folstein M, Katzman R, Price D, Stadlan EM. Clinical diagnosis of Alzheimer's disease: report of the NINCDSADRDA Work Group under the auspices of Department of Health and Human Services Task Force on Alzheimer's Disease. Neurology 1984;34: 939-944.

12. Carpenter CR, Bassett ER, Fischer GM, Shirshekan J, Galvin JE, Morris JC. Four sensitive screening tools to detect cognitive dysfunction in geriatric emergency department patients: brief Alzheimer's Screen, Short Blessed Test, Ottawa 3DY, and the Caregiver-completed AD8. Acad Emerg Med 2011;18:374-384.

13. Lee D, Yoon J, Lee K, Jhoo J, Kim K, Lee J, et al. Reliability and Validity of the Korean Version of Short Blessed Test (SBT-K) as a Dementia Screening Instrument. J Korean Neuropsychiatr Association 1999;38:13621375.

14. Lee JH, Lee KU, Lee DY, Kim KW, Jhoo JH, Kim JH, et al. Development of the Korean version of the Consortium to Establish a Registry for Alzheimer's Disease Assessment Packet (CERAD-K): clinical and neuropsychological assessment batteries. J Gerontol B Psychol Sci Soc Sci 2002;57:P47-P53.

15. Yoo S, Kim Y, Noh J, Oh K, Kim C, Namkoong K. Validity of Korean version of the Mini-International Neruopsychiatric Interview. Anxiety Mood 2006;2:50-55.

16. Kim JY, Park JH, Lee JJ, Huh Y, Lee SB, Han SK, et al. Standardization of the Korean version of the geriatric depression scale: reliability, valid- 
ity, and factor structure. Psychiatry Investig 2008;5:232-238.

17. Scheltens P, Launer L, Barkhof F, Weinstein H, van Gool W. Visual assessment of medial temporal lobe atrophy on magnetic resonance imaging: interobserver reliability. J Neurol 1995;242:557-560.

18. Koedam EGE, Lehmann M, van der Flier W, Scheltens P, Pijnenburg YL, Fox N, et al. Visual assessment of posterior atrophy development of a MRI rating scale. Eur Radiol 2011;21:2618-2625.

19. Fazekas F, Barkhof F, Wahlund LO, Pantoni L, Erkinjuntti T, Scheltens $\mathrm{P}$, et al. CT and MRI rating of white matter lesions. Cerebrovasc Dis 2002; 13(suppl 2):31-36.

20. Gianotti LRR, Künig G, Lehmann D, Faber PL, Pascual-Marqui RD, Kochi K, et al. Correlation between disease severity and brain electric LORETA tomography in Alzheimer's disease. Clin Neurophysiol 2007;
118:186-196.

21. Zion-Golumbic E, Golan T, Anaki D, Bentin S. Human face preference in gamma-frequency EEG activity. Neuroimage 2008;39:1980-1987.

22. Elgendi M, Vialatte F, Cichocki A, Latchoumane C, Jeong J, Dauwels J. Optimization of EEG frequency bands for improved diagnosis of Alzheimer disease. Conf Proc IEEE Eng Med Biol Soc 2011;2011:60876091.

23. Moe KE, Larsen LH, Prinz PN, Vitiello MV. Major unipolar depression and mild Alzheimer's disease: differentiation by quantitative tonic REM EEG. Electroencephalogr Clin Neurophysiol 1993;86:238-246.

24. Brinkmeyer J, Grass-Kapanke B, Ihl R. EEG and the Test for the Early Detection of Dementia with Discrimination from Depression (TE4D): a validation study. Int J Geriatr Psychiatry 2004;19:749-753. 